\title{
Weak side of strong topological insulators
}

\author{
Björn Sbierski, Martin Schneider, and Piet W. Brouwer \\ Dahlem Center for Complex Quantum Systems and Fachbereich Physik, Freie Universität Berlin, 14195 Berlin, Germany
}

(Received 10 February 2016; published 11 April 2016)

\begin{abstract}
Strong topological insulators may have nonzero weak indices. The nonzero weak indices allow for the existence of topologically protected helical states along line defects of the lattice. If the lattice admits line defects that connect opposite surfaces of a slab of such a "weak-and-strong" topological insulator, these states effectively connect the surface states at opposite surfaces. Depending on the phases accumulated along the dislocation lines, this connection results in a suppression of in-plane transport and the opening of a spectral gap or in an enhanced density of states and an increased conductivity.
\end{abstract}

DOI: 10.1103/PhysRevB.93.161105

Introduction. Band insulators come in topologically distinct classes, where the topologically nontrivial classes have extended surface states, which are robust to small deformations of the Hamiltonian [1-6]. The topological classification of generic band insulators in three dimensions distinguishes "strong" and "weak" topological indices [5,7]. A nonzero value of the strong index signifies a "strong topological insulator;" Surface states of strong insulators have a spectrum with an odd number of Dirac cones, and they are robust to disorder or other perturbations that break the lattice translation symmetry. In a "weak topological insulator," i.e., if the strong invariant is trivial and the weak invariant is nontrivial, the lattice translation symmetry is essential for the protection of the surface states, although, as was pointed out in a seminal article by Ringel et al. [8], the surface states of a weak topological insulator show a remarkable robustness in the presence of perturbations that preserve the lattice translation symmetry on the average $[9,10]$.

An important property of insulators with nontrivial weak indices is that a line dislocation may have topologically protected helical states, similar to the helical edge states of a two-dimensional topological insulator $[11,12]$. The precise conditions for the existence of such strongly protected states depends on the Burgers vector $\mathbf{b}$ of the dislocation $[12,13]$. The helical states along the dislocation line remain topologically protected as long as the notion of a separate dislocation with a well-defined Burgers vector remains valid. The presence of nonzero weak and strong indices is not mutually exclusive, and it is possible that a band insulator is at the same time a weak topological insulator and a strong topological insulator. Such a scenario is expected to be relevant, e.g., for BiSb compounds or for the putative Kondo topological insulator $\mathrm{SmB}_{6}$ [14]. In principle, such a "weak-and-strong topological insulator" combines an odd number of Dirac cones in the surface-state spectrum with topologically protected helical states along lattice defects.

Realistic topological insulators are often layered materials, and flakes of such materials are usually investigated in a quasitwo-dimensional slab geometry, in which the slab thickness is large enough that surface states at the bottom and top surfaces remain well separated. The presence of dislocation lines that connect the top and bottom surfaces of a weak-and-strong topological insulator, as shown schematically in Fig. 1(a), may, however, provide a mechanism by which the two surfaces are coupled nevertheless. As we show here, a finite density of dislocation lines may lead to the opening of a gap in the surface-state spectrum of a slab and to a strong suppression of electron transport parallel to the surface, although the precise scenario depends on the phase that electrons accumulate along the dislocation line. The possibility of a coupling of surface states at bottom and top surfaces via dislocation lines presents a "weak side" of topological insulators with nontrivial strong and weak indices; it does not exist for strong topological insulators with trivial weak indices, for which dislocation lines do not carry protected helical states. We now proceed with a description of our results.

Description of dislocation line defect in terms of a $\pi$-flux line. The weak indices $v_{j}=0,1, j=1,2,3$, of a topological insulator are defined with respect to a basis $\left(\mathbf{G}_{1}, \mathbf{G}_{2}, \mathbf{G}_{3}\right)$ of reciprocal lattice vectors. Together they uniquely define a reciprocal lattice vector $\mathbf{M}=(1 / 2)\left(v_{1} \mathbf{G}_{1}+v_{2} \mathbf{G}_{2}+v_{3} \mathbf{G}_{3}\right)$ [12]. As shown by Ran, Zhang, and Vishwanath, a lattice dislocation binds an odd number of helical modes if and only if its Burgers vector b satisfies [12]

$$
e^{i \mathbf{b} \cdot \mathbf{M}}=-1 \text {. }
$$

In that case, there is an odd number of surface-state Dirac cones within which electrons pick up a phase $\pi$ upon going around the position $\mathbf{r}_{\mathrm{d}}$ at which the dislocation line pierces the surface. The low-energy Dirac Hamiltonian for such surface states is accordingly

$$
H=v(\mathbf{p}+e \mathbf{A} / c) \cdot \boldsymbol{\sigma},
$$

where $v$ is the surface-state velocity, $\mathbf{p}=\left(p_{x}, p_{y}\right), \boldsymbol{\sigma}=$ $\left(\sigma_{x}, \sigma_{y}\right)$, and $\mathbf{A}(\mathbf{r})$ is the vector potential corresponding to a flux line with flux $h c / 2 e$ at position $\mathbf{r}_{\mathrm{d}}$, a " $\pi$-flux [13]." Since the total number of Dirac cones in the surface-state spectrum is odd if the strong index $v_{0}=1$, the number of surface cones described by a Dirac Hamiltonian without $\pi$-flux line is even if $\mathbf{b} \cdot \mathbf{M}$ is an odd multiple of $\pi$ [12]. For simplicity we focus on the minimal model, in which there is a single surface state with low-energy effective Hamiltonian (2) in the vicinity of a dislocation line for which the condition (1) holds.

To elucidate the relation between the surface states and the helical states propagating along the dislocation line, it is instructive to analyze the eigenstates of the Hamiltonian (2) at energy $\varepsilon=\hbar v k$ using polar coordinates $(r, \theta)$. We choose the $\pi$-flux line-the location where the dislocation line pierces the surface - as the origin. This is a problem that previously was considered in the context of graphene $[15,16]$. With the choice 
(a)

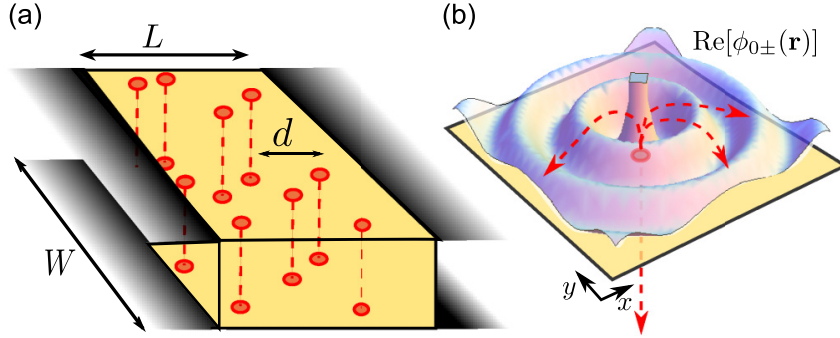

FIG. 1. (a) Topological insulator slab of size $L \times W$, with top and bottom surfaces connected via randomly placed dislocation lines with mean distance $d$. Ideal contacts are attached to the left and right, for top and bottom surfaces separately. (b) Zero-angular-momentum $(m=0)$ radial waves for nonzero wave number $k$ at the surface of the topological insulator are transmitted perfectly into and out of the one-dimensional helical states along the dislocation line.

$\mathbf{A}=(\hbar / 2 e r) \mathbf{e}_{\theta}$, where $\mathbf{e}_{\theta}$ is the unit vector for the azimuthal angle, the Hamiltonian (2) is invariant under rotations, so that we can look for eigenstates of the total angular momentum $j_{z}=l_{z}+(\hbar / 2) \sigma_{z}$. These have the form

$$
\psi_{m}(\mathbf{r})=\left(\begin{array}{c}
e^{i(m-1) \theta} \phi_{m+}(r) \\
e^{i m \theta} \phi_{m-}(r)
\end{array}\right),
$$

where $m$ is an integer and the radial wave functions $\phi_{m \pm}(r)$ satisfy

$$
\left(\partial_{r} \pm \frac{m \pm 1 / 2}{r}\right) \phi_{m \mp}(r)=i k \phi_{m \pm}(r) .
$$

For generic $m$ there is a single regular solution of Eq. (4), which describes the scattering of radial waves off the flux line. An exception is the case $m=0$, for which there are $t w o$ linearly independent solutions

$$
\phi_{0 \pm}(r)=\alpha_{\mathrm{out}} \frac{e^{i k r}}{\sqrt{r}} \pm \alpha_{\mathrm{in}} \frac{e^{-i k r}}{\sqrt{r}},
$$

for which the amplitudes $\alpha_{\text {out }}$ and $\alpha_{\text {in }}$ of outgoing and incoming radial waves can be freely chosen. Since timereversal symmetry rules out backscattering for the $m=0$ states and for the helical states propagating along the defect line [17], the incoming $m=0$ mode must be fully transmitted into the outgoing defect state, and the incoming defect state is fully transmitted into the outgoing $m=0$ mode, as shown schematically in Fig. 1(b).

Surface states in the presence of dislocation lines. We now consider transport properties and density of states of surface states for a slab geometry with multiple dislocation lines, piercing the top and bottom surfaces at random positions, see Fig. 1(a). We choose coordinates such that the bottom and top surfaces are parallel to the $x y$ plane, with transport taking place in the $x$ direction. For simplicity we take the dislocation lines to pierce bottom and top surfaces at the same in-plane position $\mathbf{r}_{\mathrm{d}}=\left(x_{\mathrm{d}}, y_{\mathrm{d}}\right)$, an assumption that is appropriate for a low-energy, long-wavelength description of a thin slab. The in-plane dimensions of the slab are $L \times W$, and we assume that the slab thickness is sufficient large, so that surface states at the bottom and top surfaces do not overlap in the absence of lattice dislocations. We take periodic boundary conditions in the $y$ direction, choosing the aspect ratio $W / L$ large enough that the results of our calculation do not depend on this choice of boundary conditions.

We calculate the density of states and the transport properties of the surface states using a scattering approach. The scattering matrix $S_{\sigma, \sigma^{\prime}}$ links the amplitudes of incoming and outgoing waves in an "ideal" part of the two surfaces, to the left and right of a section with a finite density of dislocation lines. The indices $\sigma, \sigma^{\prime}=+1,-1$ for the top and bottom surface, respectively. Dislocation lines connect the top and bottom surfaces, so that in general $S_{\sigma, \sigma^{\prime}}$ is not block diagonal. We denote the amplitudes of incoming and outgoing waves to the left (right) of the section by vectors $a_{\mathrm{L} \sigma n}^{\text {in }}$ and $a_{\mathrm{L} \sigma n}^{\text {out }}\left(a_{\mathrm{R} \sigma n}^{\text {in }}\right.$ and $\left.a_{\mathrm{R} \sigma n}^{\text {out }}\right)$, respectively, where the index $n$ refers to the transverse momentum $q_{n}=2 \pi n / W$. With this notation, the scattering matrix $S_{\sigma, \sigma^{\prime}}$ relates outgoing and incoming waves as

$$
\left(\begin{array}{c}
a_{\mathrm{L} \sigma}^{\text {out }} \\
a_{\mathrm{R} \sigma}^{\text {out }}
\end{array}\right)=\sum_{\sigma^{\prime}= \pm} S_{\sigma, \sigma^{\prime}}\left(\begin{array}{l}
a_{\mathrm{L} \sigma^{\prime}}^{\text {in }} \\
a_{\mathrm{R} \sigma^{\prime}}^{\text {in }}
\end{array}\right) .
$$

Each component $S_{\sigma, \sigma^{\prime}}$ can be decomposed into transmission and reflection blocks in the standard way,

$$
S_{\sigma, \sigma^{\prime}}=\left(\begin{array}{cc}
r_{\sigma, \sigma^{\prime}} & t_{\sigma, \sigma^{\prime}}^{\prime} \\
t_{\sigma, \sigma^{\prime}} & r_{\sigma, \sigma^{\prime}}^{\prime}
\end{array}\right) \text {. }
$$

Our strategy will be to first calculate the scattering matrix $\tilde{S}$ for a "short" slab of length $\delta L$ with only a pair of dislocation lines, and then calculate the scattering matrix $S$ of a slab of full length $L$ by concatenating scattering matrices of individual slices [18], see Fig. 2 (top). We place a pair of dislocation lines at $\mathbf{r}_{\mathrm{d}, 1}=\left(x_{\mathrm{d}}, y_{\mathrm{d}, 1}\right)$ and $\mathbf{r}_{\mathrm{d}, 2}=\left(x_{\mathrm{d}}, y_{\mathrm{d}, 2}\right)$, with $0<x_{\mathrm{d}}<\delta L$ and $0<y_{\mathrm{d}, 1}<y_{\mathrm{d}, 2}<W$ randomly chosen. Since the aspect ratio $W / L \gg 1$, the pairwise placement of dislocation in a slab (compared to placement of single dislocation lines) does not affect the in-plane conductivity or the density of states. It does, however, allow us to choose a gauge such that the vector potential $\mathbf{A}(\mathbf{r})$ is nonzero for $x=x_{\mathrm{d}}$ only,

$$
\mathbf{A}(\mathbf{r})=\frac{h}{2 e} \delta\left(x-x_{\mathrm{d}}\right) \mathbf{e}_{x} \times \begin{cases}1 & \text { if } y_{\mathrm{d}, 1}<y<y_{\mathrm{d}, 2}, \\ 0 & \text { else. }\end{cases}
$$

An important further parameter in the calculation is the phase shift $e^{i \theta_{\mathrm{d}}\left(\mathbf{r}_{\mathrm{d}}\right)}$ that electrons accumulate along the dislocation

(a)

(b)

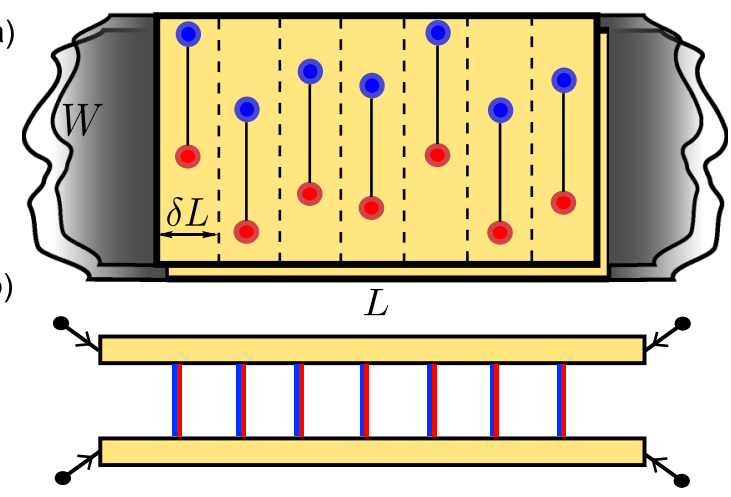

FIG. 2. Schematic picture of a top view (a) and side view (b) of the topological insulator slab. The calculational scheme involves the computation of the scattering matrix $\tilde{S}$ for a slab of width $\delta L$, followed by the concatenation of scattering matrices of individual slabs to obtain the scattering matrix $S$ of the full structure. 
line. For our calculations we found it advantageous to generalize the above procedure to slabs with an even number $2 n$ of dislocation lines.

The calculation of the scattering matrix $\tilde{S}$ for a slab with a single pair of dislocation lines turned out to be an interesting problem in its own right. Although the scattering problem for a single dislocation line is easily solved in polar coordinates, see Eq. (4), we could not find a practical way to extract a scattering matrix for the geometry of Fig. 1(a) from this solution. Instead, we compute $\tilde{S}$ from a solution of the Dirac equation for a regularized (i.e., smeared out) $\pi$ flux. (Without regularization the scattering problem with a $\pi$-flux line cannot be solved numerically.) The details of this calculation are given in the Supplemental Material [19].

Results. By concatenation of scattering matrices for slices of length $\delta L$, each with an even number $2 n$ of dislocation lines, we can construct the full scattering matrix $S$ for a slab of length $L$ with randomly placed dislocation line pairs at concentration $1 / d^{2}=N_{\mathrm{d}} / W L$, with $N_{\mathrm{d}}$ the total number of dislocation lines, see Fig. 2(a). The Landauer formula expresses the in-plane conductance $G_{\|}$and the cross conductance $G_{\perp}$ in terms of the transmission and reflection blocks of the scattering matrix $S$,

$$
G_{\|}=\frac{e^{2}}{h} \sum_{\sigma, \sigma^{\prime}= \pm 1} \operatorname{tr} t_{\sigma, \sigma^{\prime}} t_{\sigma, \sigma^{\prime}}^{\dagger}, \quad G_{\perp}=\frac{e^{2}}{h} \operatorname{tr} S_{+-} S_{+-}^{\dagger} .
$$

For the calculation of the density of states, we consider a periodic array of slabs of length $L$. In this case the spectrum of Bloch states can be obtained from the condition that

$$
S_{\kappa_{x}}(\varepsilon) \equiv\left(\begin{array}{cc}
0 & e^{-i \kappa_{x} L} \\
e^{i \kappa_{x} L} & 0
\end{array}\right) S(\varepsilon)
$$

has a unit eigenvalue, where $\hbar \kappa_{x}$ is the crystal momentum.

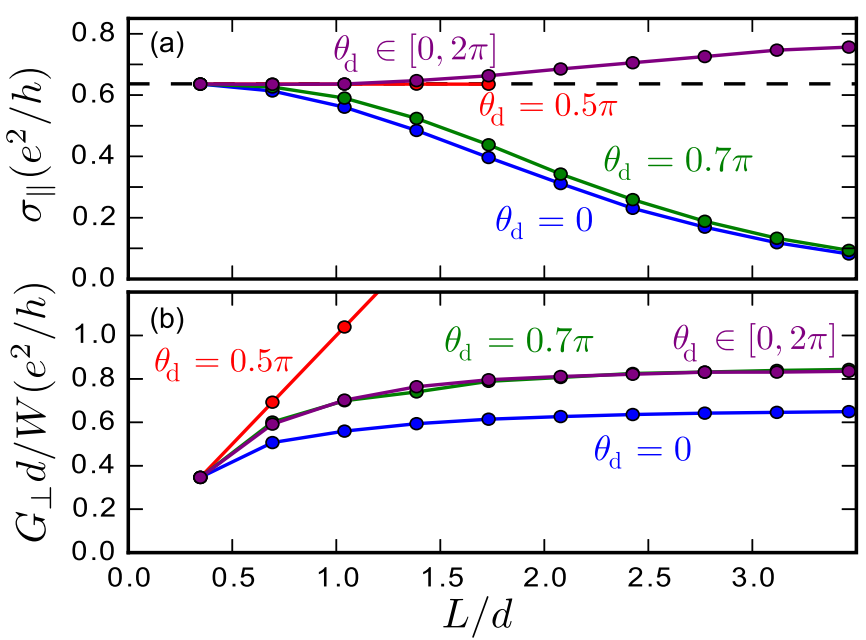

FIG. 3. Zero-energy in-plane conductivity $\sigma_{\|}$(a) and cross conductance $G_{\perp}$ (b) for a slab of weak-and-strong topological insulator with a concentration $1 / d^{2}$ of randomly placed dislocation lines. The different curves refer to different choices for the phases $\theta_{d}\left(\mathbf{r}_{d}\right)$, as shown in the figure. The dashed line in (a) denotes the clean-limit in-plane conductivity $\sigma_{\|, 0}=2 e^{2} / \pi h$. Data points denote an average over 500 disorder realizations, statistical error bars are typically smaller than the markers.
Results of the transport calculations are shown in Fig. 3 for an average over 500 random realizations of the dislocation lines. The energy $\varepsilon$ is set to zero throughout the calculation, to maximize the effect of the dislocation lines. The sample length $L$ is measured in units of the mean distance $d$ between dislocation lines, which is the only fundamental length scale in the system at zero energy. The trivial $W$ dependence of $G_{\|}$ is eliminated by considering the in-plane conductivity $\sigma_{\|}=$ $G_{\|} L / W$. For $L / d \rightarrow 0$ we recover the clean-limit conductivity $\sigma_{\|, 0}=2 e^{2} / \pi h$ of a pair of decoupled topological-insulator surfaces [20,21]. Anticipating a proportionality $G_{\perp} \propto W / d$, in Fig. 3(b) we show $G_{\perp} d / W$ as a function of $L / d$. Unlike the longitudinal conductivity $\sigma_{\|}$, the cross conductance vanishes in the clean limit $L / d \rightarrow 0$.

We observe that the in-plane conductivity has a strong dependence on the phase that electrons pick up while traveling along the dislocation lines. In particular, if all phases are equal, $\theta_{\mathrm{d}}\left(\mathbf{r}_{\mathrm{d}}\right)=\theta_{\mathrm{d}}$ for all $\mathbf{r}_{\mathrm{d}}, \sigma_{\|}$is strongly suppressed for $L \gtrsim d$ except for $\theta_{\mathrm{d}}= \pm \pi / 2$, for which we find that $\sigma_{\|}$is independent of $L / d$ within numerical accuracy [22] Figure 3 shows the representative cases $\theta_{\mathrm{d}}=0,0.7 \pi$, and $\pi / 2$, and we also present the case $0 \leqslant \theta_{\mathrm{d}}\left(\mathbf{r}_{\mathrm{d}}\right)<2 \pi$ uniformly distributed, which shows a slight increase of $\sigma_{\|}$with $L / d$. The $\theta_{\mathrm{d}}$ dependence of the cross conductance is not as strong; $\theta_{\mathrm{d}}$ mainly determines the value at which $G_{\perp}$ saturates for large $L / d$. An exception is $\theta_{\mathrm{d}}=\pi / 2$, for which we could not observe a saturation for the system sizes we could achieve.

Results for the density of states are shown in Fig. 4, again for four representative choices of the phase shifts $\theta_{\mathrm{d}}\left(\mathbf{r}_{\mathrm{d}}\right)$. For fixed $\theta_{\mathrm{d}}\left(\mathbf{r}_{\mathrm{d}}\right)=0$ we observe one or two gaps placed asymmetrically around $\varepsilon=0$. For generic fixed $\theta\left(\mathbf{r}_{\mathrm{d}}\right)=\theta_{\mathrm{d}}$ (such as the case $\theta_{\mathrm{d}}=0.7 \pi$ shown in the figure) we observe an asymmetric gap around $\varepsilon=0$. For $\theta_{\mathrm{d}}\left(\mathbf{r}_{\mathrm{d}}\right)=\pi / 2$ a symmetric gap is restored, but with one midgap state at $\varepsilon=0$ per dislocation line. Finally, for random $0 \leqslant \theta_{\mathrm{d}}\left(\mathbf{r}_{\mathrm{d}}\right)<2 \pi$ the gap is closed and the density of states near $\varepsilon=0$ is essentially constant. The gap sizes and the occurrence of states at energy $\varepsilon=0$ can be heuristically

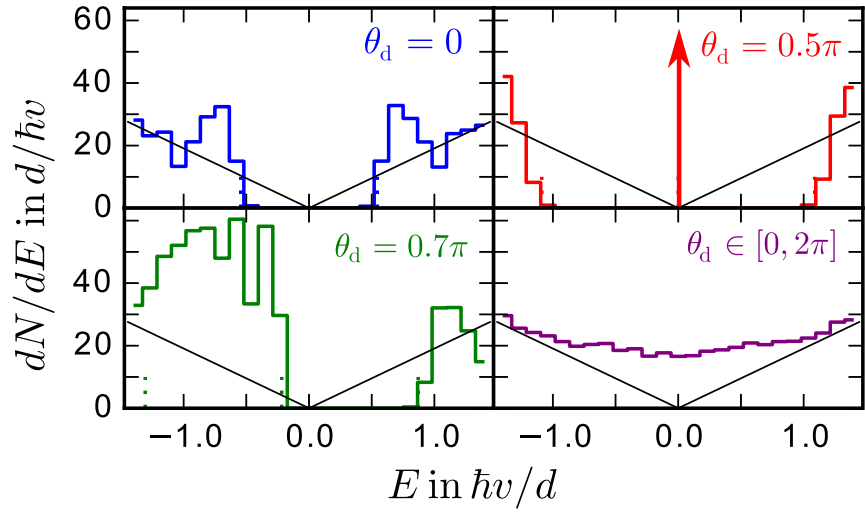

FIG. 4. Density of states $d N / d E$ of a sample with dislocation line density $1 / d^{2}$. The four curves represent the four representative scenarios for the choice of the phase shifts $\theta_{\mathrm{d}}\left(\mathbf{r}_{\mathrm{d}}\right)$, as explained in the text. The vertical dashed lines correspond to energies calculated from Eq. (11). The thin black lines denote the ideal surface-state density of states without dislocation lines. The arrow represents a Dirac delta function at zero energy. Data points denote an average over five disorder realizations and 80 values of the crystal momentum $\kappa_{x}$. 
explained by inspecting the phase matching condition for periodic trajectories traveling between the two surfaces at two neighboring dislocation lines at positions $\mathbf{r}_{\mathrm{d}}$ and $\mathbf{r}_{\mathrm{d}}^{\prime}$. Including the Berry phase $\pi$ for two-dimensional Dirac particles, this phase matching condition reads

$$
\frac{2 \varepsilon}{\hbar v}\left|\mathbf{r}_{\mathrm{d}, i}-\mathbf{r}_{\mathrm{d}, j}\right|+\theta_{\mathrm{d}}\left(\mathbf{r}_{\mathrm{d}}\right)+\theta_{\mathrm{d}}\left(\mathbf{r}_{\mathrm{d}}^{\prime}\right)+\pi=0 \quad(\bmod 2 \pi) .
$$

Setting $\left|\mathbf{r}_{\mathrm{d}}-\mathbf{r}_{\mathrm{d}}^{\prime}\right|=W / 2 n$, which is the largest typical distance between neighboring dislocation lines with $2 n=6$ dislocations in a slice gives a good estimate of the numerically obtained gap sizes, see Fig. 4. The absence of states around $\varepsilon=0$ indicates that pairing of more distant dislocation lines does not occur.

Conclusion. We have investigated the effects of dislocation line zero modes coupling top and bottom surfaces of a strong-and-weak topological insulator slab. Our numerical calculations based on a scattering approach reveal a rich phenomenology for transport properties and density of states depending on the phase shifts $\theta_{\mathrm{d}}\left(\mathbf{r}_{\mathrm{d}}\right)$ that electrons accumulate along the dislocation lines. For a thin, homogenous slab, a constant phase shift $\theta_{\mathrm{d}}$ for all dislocation lines can be expected to be a good approximation. Except for the special cases $\theta_{\mathrm{d}}= \pm \pi / 2$, this results in a spectral gap around zero energy and a corresponding strong suppression of in-plane transport. For a thick slab, where dislocation lines are not necessarily straight, it is conceivable that the phase shifts $\theta_{\mathrm{d}}\left(\mathbf{r}_{\mathrm{d}}\right)$ are uniformly distributed. In this case, the in-plane conductivity and the density of states at the nodal energy are enhanced by the presence of dislocation lines. In this work, we neglected the wavefunction overlap for neighboring dislocation line modes. Recently, such hybridization effects were studied in Ref. [23].

In principle, the dislocation-line-mediated coupling between the top- and bottom surfaces can be described by an effective Hamiltonian involving two Dirac cones coupled by a matrix-valued "potential." Such an effective model was considered by Mong et al. in the context of the transport through a single surface of a weak topological insulator with two (coupled) Dirac cones [9]. The same description can also be applied to the system studied here, although the two Dirac cones now refer to different surfaces. Our analysis shows that the disorder type in such a model depends strongly on the phases accumulated along the dislocation lines: While a mass term is responsible for the opening of a spectral gap (as for $\theta_{\mathrm{d}}\left(\mathbf{r}_{\mathrm{d}}\right)=\theta_{\mathrm{d}}$ constant, $\left.\theta_{\mathrm{d}} \neq \pm \pi / 2\right)$, a constant scalar potential creates the asymmetry around $\varepsilon=0$ (which we observe for generic $0 \leqslant\left|\theta_{\mathrm{d}}\right|<\pi / 2$ ), and zero-average disorder terms lead to the "flattening" of the density-of-states singularity at zero energy. Establishing a more rigorous understanding of our results in terms of a Hamiltonian theory would be a formidable task for future work.

Acknowledgments. We gratefully acknowledge financial support by the Helmholtz Virtual Institute "New states of matter and their excitations" and the Alexander von HumboldtStiftung.
[1] C. L. Kane and E. J. Mele, Phys. Rev. Lett. 95, 226801 (2005).

[2] C. L. Kane and E. J. Mele, Phys. Rev. Lett. 95, 146802 (2005).

[3] B. A. Bernevig and S.-C. Zhang, Phys. Rev. Lett. 96, 106802 (2006).

[4] J. E. Moore and L. Balents, Phys. Rev. B 75, 121306 (2007).

[5] L. Fu, C. L. Kane, and E. J. Mele, Phys. Rev. Lett. 98, 106803 (2007).

[6] R. Roy, Phys. Rev. B 79, 195322 (2009).

[7] L. Fu and C. L. Kane, Phys. Rev. B 76, 045302 (2007).

[8] Z. Ringel, Y. E. Kraus, and A. Stern, Phys. Rev. B 86, 045102 (2012).

[9] R. S. K. Mong, J. H. Bardarson, and J. E. Moore, Phys. Rev. Lett. 108, 076804 (2012).

[10] I. C. Fulga, B. van Heck, J. M. Edge, and A. R. Akhmerov, Phys. Rev. B 89, 155424 (2014).

[11] J. C. Y. Teo and C. L. Kane, Phys. Rev. B 82, 115120 (2010).

[12] Y. Ran, Y. Zhang, and A. Vishwanath, Nat. Phys. 5, 298 (2009).

[13] K.-I. Imura, Y. Takane, and A. Tanaka, Phys. Rev. B 84, 035443 (2011); R.-J. Slager, A. Mesaros, V. Juričić, and J. Zaanen, ibid. 90, 241403 (2014).

[14] Y. Ando, J. Phys. Soc. Jpn. 82, 102001 (2013).

[15] J. Heinl, M. Schneider, and P. W. Brouwer, Phys. Rev. B 87, 245426 (2013).
[16] M. Schneider and P. W. Brouwer, Phys. Rev. B 89, 205437 (2014).

[17] For the gauge chosen here, time-reversal amounts to the operation $\psi \rightarrow i \sigma_{y} \psi^{*}$, followed by a gauge transformation $\psi \rightarrow e^{i \theta} \psi$. This corresponds to the change $m \rightarrow-m$, so that time-reversal symmetry forbids backscattering for the $m=0$ mode only.

[18] J. H. Bardarson, J. Tworzydlo, P. W. Brouwer, and C. W. J. Beenakker, Phys. Rev. Lett. 99, 106801 (2007).

[19] See Supplemental Material http://link.aps.org/supplemental/ 10.1103/PhysRevB.93.161105 for the derivation of the scattering matrix as well as for details of the transport and density of states calculation.

[20] M. I. Katsnelson, Eur. Phys. J. B 51, 157 (2006).

[21] J. Tworzydlo, B. Trauzettel, M. Titov, A. Rycerz, and C. W. J. Beenakker, Phys. Rev. Lett. 96, 246802 (2006).

[22] In the case $\theta_{\mathrm{d}}=\pi / 2$, we observe that the scattering matrix at zero energy ceases to be unitary for $L / d \gtrsim 2$, which is the reason for the relatively small upper bound on the system sizes shown in Fig. 3. This can be understood from the perspective of bound state formation, once the number of bound states $N_{\mathrm{d}}$ is of the same order as the number of modes considered in the scattering matrix, $N_{\text {eff }}$.

[23] R.-J. Slager, V. Juricic, V. Lahtinen, and J. Zaanen, arXiv:1509.07705v2 (2015). 